\title{
miR-320b Is Down-Regulated in Psoriasis and Modulates Keratinocyte Proliferation by Targeting AKT3
}

\author{
Yan Wang, ${ }^{1,2}$ Xiaojing Yu, ${ }^{1}$ Lihua Wang, ${ }^{2}$ Weiyuan Ma, ${ }^{1}$ and Qing Sun ${ }^{1,3}$
}

\begin{abstract}
We investigated the molecular mechanisms underlying the role of miRNAs in the pathogenesis of psoriasis to discover novel potential diagnostic markers and treatment targets. We screened Chinese Han individuals using gene chip technology to identify differentially expressed miRNAs in the epidermal tissue of lesions from patients with psoriasis $v$ ersus that from healthy controls. We also used bioinformatics methods and molecular biology experiments to predict and verify target genes and signaling pathways that may have an underlying role in normal human epidermal keratinocyte (NHEK) proliferation. Differentially expressed miRNAs were found in the epidermal tissue of lesions from patients with psoriasis; 45 were upregulated, and 71 were downregulated. Among them, miR-320b was significantly downregulated. Low miR-320b expression levels in NHEKs promoted cell proliferation. The luciferase assay results showed that AKT3 is a target gene of miR-320b. The protein phosphorylation levels of STAT3 and SAPK/JNK in the intracellular signaling pathway were significantly upregulated by miR-320b downregulation. Our findings indicate that miR-320b negatively regulates NHEK proliferation by targeting AKT3 to regulate the STAT3 and SAPK/JNK signaling pathways and might participate in the pathogenesis of psoriasis in Chinese Han populations. miR-320b may also be a novel diagnostic marker or therapeutic target for this disease.
\end{abstract}

KEY WORDS: psoriasis; keratinocytes; miR-320b; AKT3 protein kinase.

\section{INTRODUCTION}

Psoriasis is a common immune-mediated, chronic inflammatory skin disease with an incidence of approximately $2-3 \%$ of the world's population [1]. The etiology and pathogenesis of this disease are unknown. Present studies show that psoriasis is a multifactorial genetic

\footnotetext{
${ }^{1}$ Department of Dermatology, Qilu Hospital, Shandong University, 107 Wenhua W Rd, Jinan, 250012, China

${ }^{2}$ Department of Dermatology, Jinan Central Hospital Affiliated to Shandong University, Jinan, 250013, China

${ }^{3}$ To whom correspondence should be addressed at Department of Dermatology, Qilu Hospital, Shandong University, 107 Wenhua W Rd, Jinan, 250012, China. E-mail: sunqing7226@163.com
}

disease related to different factors, including heredity, environment, immunity, infection, and psychological factors [2]. The pathological characteristics of psoriasis are hyperproliferation, aberrant keratinocyte differentiation, and inflammatory cell infiltration into the dermis and epidermis [3]. However, the underlying mechanisms regulating the epidermal defects and immunological dysfunction remain uncertain.

MicroRNAs (miRNAs) are a class of endogenous, non-coding RNAs that are approximately 20-25 nucleotides in length and function mainly by binding to the $3^{\prime}$ untranslated region of target gene mRNA [4, 5]. They are widely distributed in eukaryotes and are involved in many biological processes, including embryonic development, hematopoietic processes, fat metabolism, organogenesis, cell differentiation, proliferation, apoptosis, and tumorigenesis. In 
recent years, an increasing number of studies have shown that several miRNAs, such as miR-21 [6], miR-146 [7], miR-424 [8], miR-203 [9, 10], miR-125 [11], and miR197 [12], are associated with the pathology of psoriasis. One of the pathological characteristics of psoriasis is a reduction in the mitotic cycle duration in keratinocytes, and this reduction results in accelerated proliferation. Therefore, this study screened Chinese Han individuals using gene chip technology to identify differentially expressed miRNAs in the epidermal tissue of lesions from patients with psoriasis versus that from healthy controls. Bioinformatics methods and molecular biology experiments were also used to predict and verify target genes and signaling pathways that may have an underlying role in human epidermal keratinocyte (NHEK) proliferation. miR-320 is a newly discovered microRNA family that is expressed specifically in epithelial tissues and is involved in skin development, homeostasis, and functional maintenance; thus, it is an important regulatory factor in the skin [13]. miR-320b, a member of this family, was significantly downregulated in the epidermal tissue of lesions from patients with psoriasis. Therefore, we selected miR-320b to study its role in the pathogenesis of psoriasis.

In addition, this study aimed to provide a solid foundation for further study of the molecular mechanisms of miRNAs in the pathogenesis of psoriasis. These data may lead to the discovery of novel diagnostic markers and treatment targets.

\section{MATERIALS AND METHODS}

\section{Participants}

This study was approved by the Research Ethics Committee of Qilu Hospital, Shandong University (No. KYLL 2015 108) and was performed in accordance with the principles of the 1964 Declaration of Helsinki. All study participants provided informed consent. Patients were diagnosed with psoriasis according to the diagnostic criteria for psoriasis [14] and histopathological analyses at the Department of Dermatology at Qilu Hospital, Shandong University, from June 2015 to December 2015. The recruited patients did not have other types of skin diseases or other immune or neoplastic diseases. In addition, they had not received systematic treatment with glucocorticoid steroids, immunosuppressive agents, tretinoin, or other drugs during the past 3 months, and they had not received phototherapy or psoriasis treatment using external preparations within the past month. Our test group consisted of
10 Chinese Han patients with psoriasis; 3 were males, 7 were females, and the average age was 36.1 years (range 19-52 years). Our normal control (NC) group consisted of ten healthy individuals, 4 males and 6 females, with an average age of 44.4 years (range 25-63 years). Normal skin tissue samples were obtained from this cohort during plastic surgery, and lesion skin samples were obtained from lesions from the patient cohort. All sample tissues were treated with formalin and embedded in paraffin. Paraffinembedded tissues were cut into $5-10-\mu \mathrm{m}$ slices. The lesions were identified using a microscope, and the epidermal tissue was scraped with a sterile surgical blade and placed into a $1.5-\mathrm{mL}$ sterile centrifuge tube.

\section{RNA Quantification}

RNA was extracted with TRIzol (Invitrogen Life Technologies, San Diego, CA, USA) and purified using an RNeasy Mini kit (Qiagen, Hilden, Germany) according to the manufacturer's instructions. Purified RNA concentrations were measured using an ND-1000 spectrophotometer (NanoDrop, Wilmington, DE, USA), and electrophoresis was performed to assess RNA integrity.

\section{miRNA Labeling and Microarray Hybridization}

Total RNA was extracted from four patients with psoriasis and four normal controls. The miRNA was labeled with a miRCURYTM Array Power Labeling kit (Cat no. 208032-A, Exiqon, Vedbaek, Denmark), using $1 \mu \mathrm{g}$ of labeled RNA with $2 \mu \mathrm{L}$ of water and $1 \mu \mathrm{L}$ each of CIP buffer and CIP enzyme. The reaction mixtures were mixed and incubated at $37^{\circ} \mathrm{C}$ for $30 \mathrm{~min}$, followed by $95{ }^{\circ} \mathrm{C}$ for $5 \mathrm{~min}$ to terminate the reaction. Next, $3 \mu \mathrm{L}$ of the labeling buffer, $1.5 \mu \mathrm{L}$ of the fluorescent label (Hy3 $\left.3^{\mathrm{TM}}\right), 2 \mu \mathrm{L}$ of DMSO, and $2 \mu \mathrm{L}$ of the labeling enzyme were added, followed by incubation at $16^{\circ} \mathrm{C}$ for $1 \mathrm{~h}$. The reaction was stopped by incubating the samples at $65{ }^{\circ} \mathrm{C}$ for $15 \mathrm{~min}$. After labeling was complete, the samples were hybridized to a miRCURYTM LNA Array (v.18.0) microarray (Exiqon) using $25 \mu \mathrm{L}$ of the sample mixed with $25 \mu \mathrm{L}$ of hybridization buffer. Then, the samples were denatured for 2 min at $95^{\circ} \mathrm{C}$ and incubated on ice for $2 \mathrm{~min}$. Hybridization was performed with a 12-Bay Hybridization System (Nimblegen Systems, Inc., Madison, WI, USA) for 16$20 \mathrm{~h}$ at $56^{\circ} \mathrm{C}$. After hybridization, the slides were washed several times using a wash buffer kit (Exiqon) and scanned using a GenePix 4000B microarray scanner (Axon Instruments, Foster City, CA, USA). 


\section{Scanning and Data Analysis}

GenePix Pro 6.0 (Axon Instruments) was used to read the chip and scan the image and for grid alignment and data extraction. Replicated miRNAs were averaged, and miRNAs with intensities $\geq 30$ in all samples were chosen to calculate the normalization factor. The expressed data were normalized using median normalization. After normalization, significantly differentially expressed miRNAs between the two groups were identified through fold changes and $P$ values, which were evaluated twice. The fold changes were used to screen for differential miRNA expression between the two samples, and finally, hierarchical clustering was performed to identify distinguishable miRNA expression profiles among the samples.

\section{Real-Time PCR Verification of miRNAs}

Six differentially expressed miRNAs were selected for real-time PCR (RT-PCR) to verify the miRNA microarray results, and a fluorescent real-time quantitative method was used. Primer Premier 5.0 (Premier Biosoft, Palo Alto, CA, USA) was used to design primers (refer to Table 1), which were synthesized by Shanghai Kang Cheng Biological Co., Ltd. (Shanghai, China). RT-PCR was performed in 384-well plates using an ABI 7300 system (Applied Biosystems, Foster City, CA, USA). The total reaction volume was $10 \mu \mathrm{L}$, which consisted of $2 \mu \mathrm{L}$ of the corresponding cDNA template, $5 \mu \mathrm{L}$ of $2 \times$ Master Mix, $0.5 \mu \mathrm{L}$ of each PCR-specific primer $(10 \mu \mathrm{M})$, and $2.5 \mu \mathrm{L}$ of water. RT-PCR for U6 and other miRNAs was performed under the following thermocycling conditions: $95^{\circ} \mathrm{C}$ for $10 \mathrm{~min}$ and then 40 cycles at $95^{\circ} \mathrm{C}$ for $10 \mathrm{~s}$ and $60{ }^{\circ} \mathrm{C}$ for $60 \mathrm{~s}$; finally, the fluorescence intensities were measured. The specificity of the reactions was determined by melting curve analysis. The relative miRNA expression levels were calculated using the $2^{-\Delta \Delta \mathrm{CT}}$ method.

\section{Computational Prediction of miRNA Targets}

The target genes of miR-320b were predicted using two types of miRNA target gene prediction software, Miranda and TargetScan 2. These software programs respectively identified 5982 and 1573 potential target genes, of which 553 target genes, including AKT3, CMPK1, PTEN, and $R U N X 1$, were found in both analyses. $A K T 3$ was further investigated in cell proliferation experiments.

\section{Cell Culture}

NHEKs were used for the in vitro experiments. A specimen was originally obtained as leftover normal skin from a child under 6 years of age following surgery, and permission for use was provided by informed consent. After cutting the skin tissue into pieces, the minced tissue was soaked in a digestion solution containing a dispersant enzyme and type I collagenase for $60 \mathrm{~min}$ at $37^{\circ} \mathrm{C}$. Trypsin was added, and following a 30-min digestion, DNase was added for 5 min to obtain single cells. Next, Dulbecco's modified Eagle's medium (DMEM) containing 10\% fetal bovine serum (FBS) (Thermo Fisher Scientific, Waltham, MA, USA) was added to neutralize the enzymatic reactions, and the sample was passed through a $100-\mu \mathrm{m}$ cell filter. After centrifugation, the pelleted cells were cultured in DMEM containing 10\% FBS and $10 \mathrm{mmol} / \mathrm{L}$ ROCK protein kinase inhibitor, which was replaced 3 days later with fresh CnT07 medium (Premedical Laboratories Co., Ltd., Beijing, China). The purified NHEKs were obtained after 2-3 passages, and the third passage of NHEKs was used for the subsequent experiments.

\section{Quantitative PCR to Measure miR-320b Expression in NHEKs}

Cells were harvested from 6-well plates at $80 \%$ confluency and centrifuged at $760 \times g$ for $5 \mathrm{~min}$. The supernatant was discarded, and RNA was extracted from the cell pellet using a Tripol kit (Cat no. 3101-100, Shanghai Pufei Biotech Co., Ltd., Shanghai, China). Complementary DNA was obtained by reverse transcription using an M-MLV kit (Cat no. M1705, Promega, Madison, WI, USA) and reverse transcription primers from Guangzhou Rui Bo Biological Technology Co., Ltd. (Guangzhou, China). U6 was used as a reference gene to normalize the RT-PCR data, and the data were analyzed by the $2^{-\Delta \Delta \mathrm{CT}}$ method.

\section{Transfection}

Lentiviral transfection was used to downregulate the expression of miRNA-320b in NHEKs, and changes in cell proliferation were observed. NHEKs were divided into a miR-320b-kD (DOWN) group and a normal control (NC) group. The lentiviral vectors (HSA-MIR-320B-Sponges and GV369) and the target sequence (5'-TTGC CCTCTCGACCAGCTTTTCTTCTTGCCCTCTCGAC CAGCTTTTCTTCTTGCCCTCTCGACCAGCT TTTCCGTATTGCCCTTCTGCCCTTCTLCCCTTCTG CCCTCTCGACCAGCTTTTCTTCTTGCCCTC TCGACCAGCTTTTCTTC-3') were designed and prepared by Shanghai Genechem Co. Ltd. (Shanghai, China). 
Table 1. Primer Sequences used for Real-Time PCR for miRNA Verification

\begin{tabular}{ll}
\hline Gene & Primer sequence for reverse transcription \\
\hline$U 6$ & 5'-CGCTTCACGAATTTGCGTGTCAT-3' \\
has-let-7d-3p & 5'-GTCGTATCCAGTGCGTGTCGTGGAGTCGGCAATTGCACTGGATACGACAGAAAG-3' \\
hsa-miR-30d-5p & 5'-GTCGTATCCAGTGCGTGTCGTGGAGTCGGCAATTGCACTGGATACGACCTTCCAG-3' \\
has-let-7g-5p & 5'-GTCGTATCCAGTGCGTGTCGTGGAGTCGGCAATTGCACTGGATACGACAACTGTA-3' \\
hsa-miR-320b & 5'-GTCGTATCCAGTGCGTGTCGTGGAGTCGGCAATTGCACTGGATACGACTTGCCC-3' \\
hsa-miR-1246 & 5'-GTCGTATCCAGTGCGTGTCGTGGAGTCGGCAATTGCACTGGATACGACCCTGCT-3' \\
hsa-miR-4475 & 5'-GTCGTATCCAGTGCGTGTCGTGGAGTCGGCAATTGCACTGGATACGACATAATG-3' \\
\hline
\end{tabular}

\section{3-(4,5-Dimethylthiazol-2-yl)-2,5-Diphenyltetrazolium Bromide Assay and Bromodeoxyuridine Staining}

The in vitro growth of NHEKs $\left(5 \times 10^{3}\right.$ cells/well $)$ was measured in a 96-well plate using 3-(4,5-dimethylthiazol-2-yl)-2,5-diphenyltetrazolium bromide (MTT) assays. For each time point, three wells were seeded for each group, followed by a $24-\mathrm{h}$ incubation at $37^{\circ} \mathrm{C}$ and $5 \% \mathrm{CO}_{2}$ for cell adhesion. The assays were performed for each group at $24 \mathrm{~h}$ (day 1), $48 \mathrm{~h}$ (day 2), $72 \mathrm{~h}$ (day 3), and $96 \mathrm{~h}$ (day 4 ) by adding $20 \mu \mathrm{L}$ of MTT $(5 \mathrm{mg} / \mathrm{mL})$ per well. The plates were incubated for $4 \mathrm{~h}$ at $37^{\circ} \mathrm{C}$ and $5 \% \mathrm{CO}_{2}$ and then centrifuged $(2000 \times g)$ for $5 \mathrm{~min}$. The supernatants were discarded, and $100 \mu \mathrm{L}$ of DMSO was added per well to terminate the reaction. The optical density (OD) of each well was measured using a spectrophotometer at $490 \mathrm{~nm}$.

Further examination of in vitro cell proliferation was performed using a bromodeoxyuridine (BrdU) kit (cat. no. 11647229001, Roche, Basel, Switzerland) according to the manufacturer's instructions.

\section{Dual-Luciferase Reporter Assay}

The miR-320b binding site of AKT3 was predicted by TargetScan software (available at http:// www.targetscan.org). Mutated miR-320b binding site (pMIR-REPORT Luciferase-AKT3-3'UTR (MT)) and wild-type binding site (pMIR-REPORT LuciferaseAKT3-3'UTR (WT)) sequences of AKT3 were inserted into a reporter plasmid. Experimental plasmids (pMIRREPORT Luciferase-AKT3-3'UTR (WT)), Obio Bio (H9684; pMIR-REPORT Luciferase-AKT3-3'UTR (MT)), and Obio Bio (H9685) were constructed and sequenced by Obio Technology Corp., Ltd. (Shanghai). Lipofectamine 2000 (Invitrogen) was used to co-transfect wild-type or mutant dual reporter vectors with miR-320b mimics in 293 T cells (Chinese Academy of Sciences Cell Bank,China). The cells were cultured after transfection. After $48 \mathrm{~h}$, the luciferase activity was measured using a dual-luciferase reporter assay system (Promega, USA) according to the manufacturer's instructions.

\section{Western Blot Analysis}

Following transfection, cells from the KD and NC groups were washed twice with Dulbecco's phosphatebuffered saline (DPBS) (Cat. no 14287072, Thermo Fisher Scientific), and then pre-cooled $2 \times$ lysis buffer was added. The cells were scraped off and transferred into micro test EP tubes (Eppendorf, Hamburg, Germany) and kept on ice for 10-15 min, followed by sonication consisting of four 5-s pulses at $200 \mathrm{~W}$, followed by a 2 -s interval between pulses. The sonicated samples were centrifuged $(12,000 \times g)$ at $4{ }^{\circ} \mathrm{C}$ for $15 \mathrm{~min}$, and the protein concentrations were measured by the supernatant BCA method with a BCA protein assay kit (Cat no. P0010S, Beyotime Biotechnology, Haimen, China). The protein concentration of each sample was adjusted to $2 \mu \mathrm{g} / \mu \mathrm{L}$, and sodium dodecyl sulfate-polyacrylamide gel electrophoresis was performed. The proteins were then transferred onto polyvinylidene difluoride (PVDF) membranes. The PVDF membranes were blocked (room temperature) with a Tris-buffered saline-Tween 20 (TBST) solution containing 5\% skimmed milk at $25^{\circ} \mathrm{C}$ and then incubated with a rabbit monoclonal antibody diluted at 1:2000 (ab32505, Abcam, Cambridge, United Kingdom) at $4{ }^{\circ} \mathrm{C}$ overnight, followed by incubation with an enzyme-labeled secondary rabbit IgG antibody at a dilution of 1:5000 at room temperature for $1 \mathrm{~h}$ (sc-2004, Santa Cruz Biotechnology, Inc., Dallas, TX, USA). Visualization was performed using Xray autoradiography and a Pierce ${ }^{\mathrm{TM}}$ ECL Western blotting substrate kit (Thermo Fisher Scientific, USA) according to the manufacturer's instructions.

Fig. 1. Differential expression of miRNAs in psoriasis lesions and normal tissue. Differential expression of miRNAs in the epidermal tissue from patients with psoriasis $(n=4)$ and normal controls $(n=4)$. Red indicates high relative expression and green indicates low relative expression. miRNA with expression fold change $>2$ and with FDR $<0.05$ was considered statistically significant. 


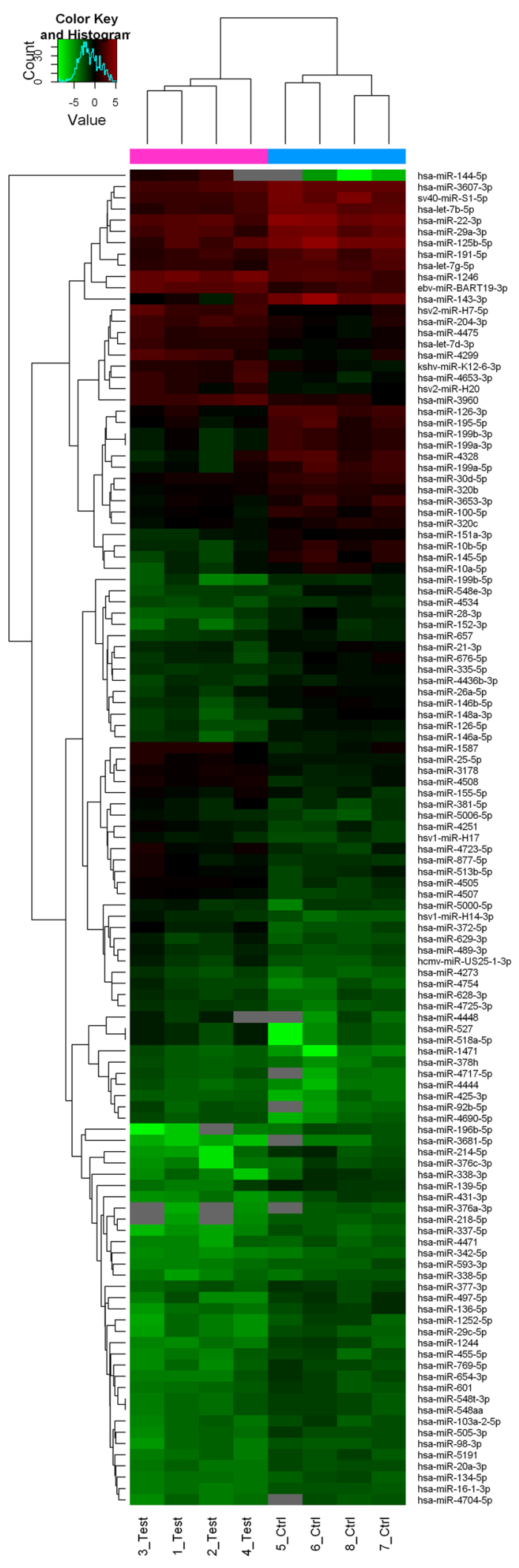




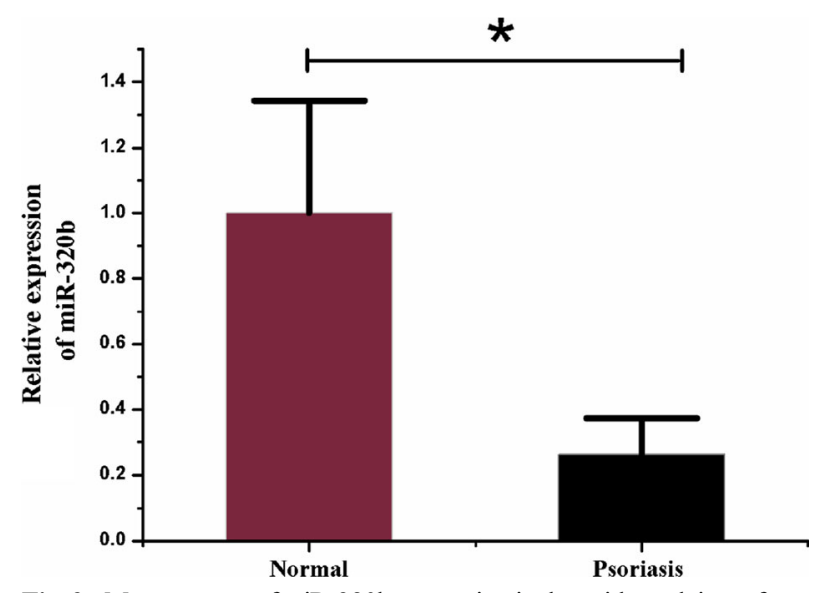

Fig. 2. Measurement of miR-320b expression in the epidermal tissue from patients with psoriasis $(n=6)$ and normal controls $(n=6)$ by qRT-PCR assay. Compared to control group, miR-320 expression in lesions of psoriasis vulgaris was significantly downregulated. qRT-PCR real-time quantitative fluorescence polymerase chain reaction; *comparison between the two groups, $P<0.05$.

\section{PathScan}

A PathScan® antibody array kit (Cell Signaling Technology Danvers, MA, USA) was used to detect and compare changes in the signal transduction of important signaling molecules between cells from the KD and NC groups. After removing the culture solution, the cells were washed 1-2 times with ice-cold PBS, followed by the addition of $1 \times$ cell lysis buffer with $1 \mathrm{mM}$ phenylmethylsulfonyl fluoride. The cells were placed on ice for $2 \mathrm{~min}$, and the concentration range was adjusted to $0.2-1.0 \mathrm{mg} / \mathrm{mL}$ with the array dilution buffer before use. For PathScan $\AA$ array detection, $100 \mu \mathrm{L}$ of blocking solution was added to each well and incubated at $25^{\circ} \mathrm{C}$ for $15 \mathrm{~min}$. Following the removal of the blocking solution, $50-75 \mu \mathrm{L}$ of cell lysis samples was added to each well and incubated at $25^{\circ} \mathrm{C}$ for $2 \mathrm{~h}$ or $4{ }^{\circ} \mathrm{C}$ overnight. The

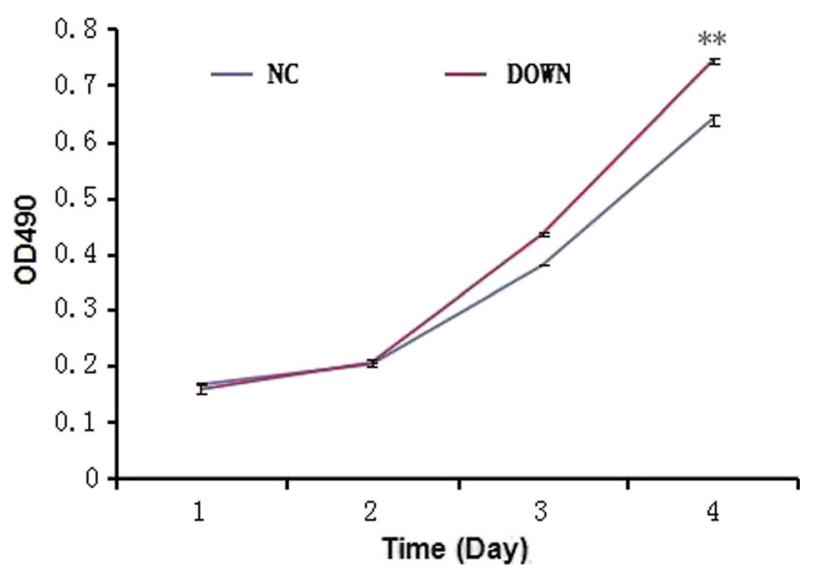

Fig. 4. Cell proliferation test by MTT. NHEKs were transfected by lentivirus and divided into a miR-320b-KD (DOWN) group and a normal control (NC) group. Cell proliferation was measured by MTT assay $96 \mathrm{~h}$ after transfection. Data were analyzed using Student's $t$ test. $* P<0.05$; $* * P<0.01$.

wells were washed three times for $5 \mathrm{~min}$, each time with $1 \times$ array cleaning solution, followed by the addition of $75 \mu \mathrm{L}$ of $1 \times$ antibody test mixture solution and a $1-h$ incubation. Next, the wells were washed four times for $5 \mathrm{~min}$, each time with $1 \times$ array cleaning solution. Following a 30-min incubation with $75 \mu \mathrm{L}$ of $1 \times$ HRP-linked streptavidin and four additional washes using the $1 \times$ array cleaning solution, the device was disassembled, and the slide was immersed in a $1 \times$ cleaning solution. Imaging was performed using a chemiluminescence imaging system (CLINX ChemiScope 5300).

\section{Data Analysis}

SPSS statistical software version 19.0 (IBM Corp., Armonk, NY, USA) was used for data analysis and

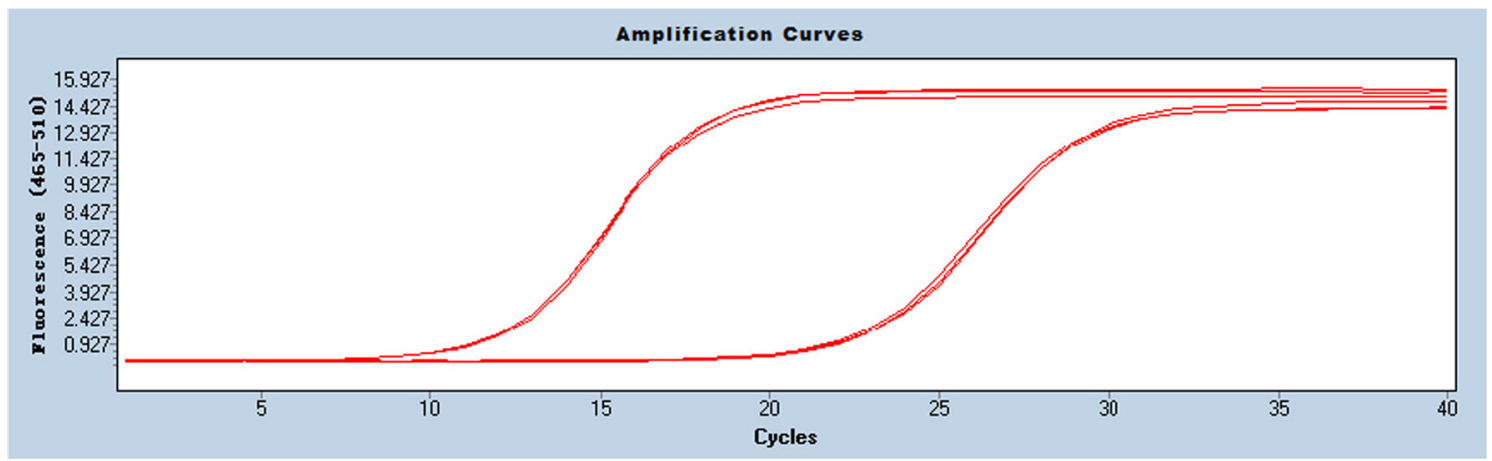

Fig. 3. Mir-320b expression in NHEKs detected by qRT-PCR. The amplification curve shows a single peak, demonstrating there is no non-specific amplification. 
statistical comparisons. The data are presented as the mean \pm standard deviation (mean \pm SD) unless otherwise stated, and a $t$ test for two independent samples was performed for comparisons between the experimental and control groups. $P<0.05$ was considered statistically significant.

\section{RESULTS}

\section{Identification of Multiple Aberrantly Expressed miRNAs in the Epidermal Tissue of Lesions from Patients with Psoriasis}

The threshold criteria of a fold change $>2$ or $<0.5$ and a $P$ value $\leq 0.05$ revealed 116 differentially expressed miRNAs in the epidermal tissues of four patients with psoriasis compared to those found in four healthy controls. Of these differentially expressed miRNAs in the epidermal tissue of lesions from patients with psoriasis, 45 were upregulated, and 71 were downregulated (Fig. 1).

\section{miR-320b Expression Was Down-Regulated in Epi- dermal Tissue Lesions in Psoriasis}

We found that miR-320b expression levels were significantly lower in epidermal tissue lesions in patients with psoriasis than in normal control tissue (fold change of 0.497, $P=0.0034)$. Quantitative reverse transcription PCR (qRT-PCR) was used to measure miR-320b expression in the epidermal tissues of six patients and six normal controls. We verified that miR-320b expression levels were significantly lower in the epidermis of patients with psoriasis than in the epidermis of normal controls $(P=0.0005$; Fig. 2).

\section{Increased Hsa-miR-320b Expression in NHEKs}

According to quantitative PCR results with $U 6$ as an internal reference for standardization, hsa-miR-320b was highly expressed in NHEKs (Fig. 3).

\section{Hsa-miR-320b Inhibits Keratinocyte Proliferation}

Next, cell viability was measured by MTT assays at $24,48,72$, and $96 \mathrm{~h}$ after transfection. We found that keratinocyte proliferation was significantly higher in the $\mathrm{KD}$ group than in the $\mathrm{NC}$ group $(P<0.0015$; Fig. 4). Furthermore, after 3 days of transfection, cell viability was evaluated using BrdU incorporation on day 4. The results also showed that keratinocyte proliferation was significantly higher in the $\mathrm{KD}$ group than in the $\mathrm{NC}$ group $(P<0.05$; Fig. 5).
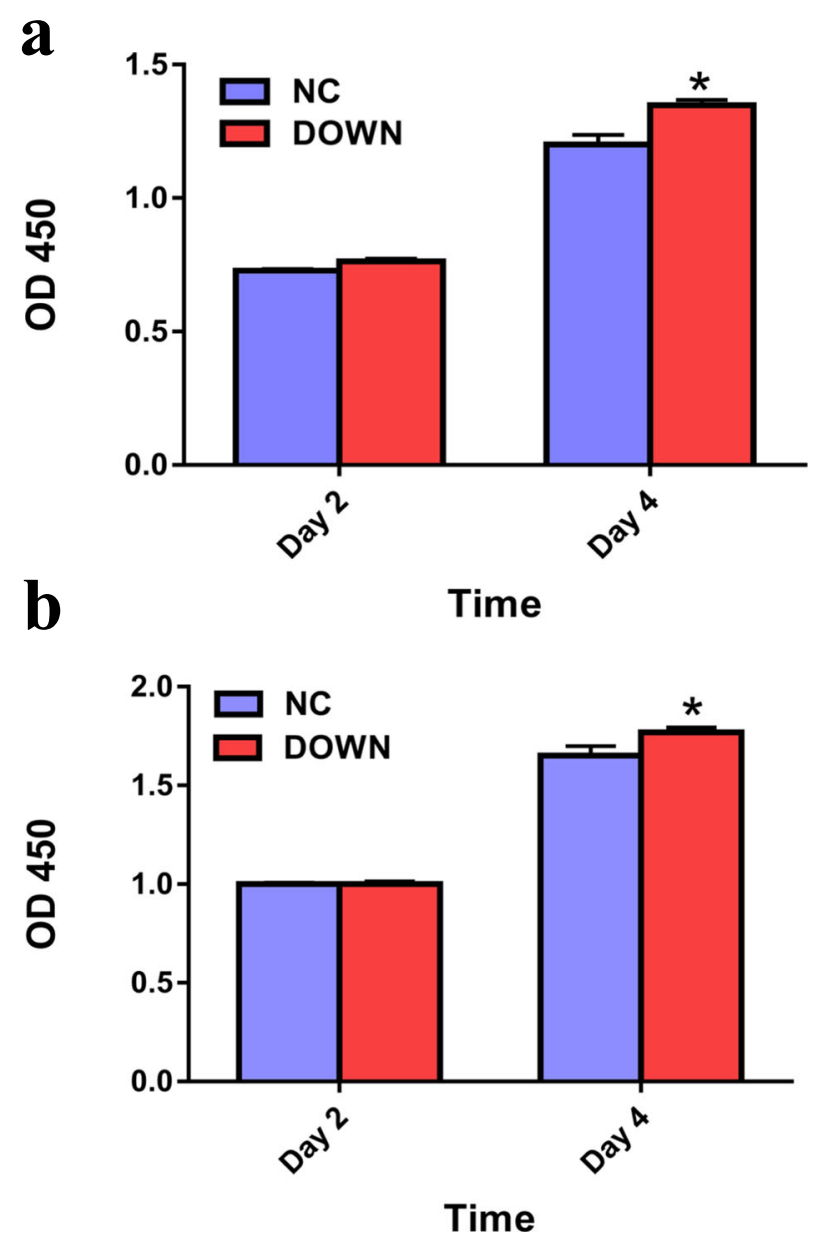

Fig. 5. Cell proliferation detected by BrdU. The proliferation viability of primary keratinocytes in the miR-320b-knockdown (down) group was higher than that in the normal control (NC) group at day $4(P<0.05)$. Histograms show plots of a OD450 and b OD450/fold change versus time. $* P<0.05$.

\section{Hsa-miR-320b Targets AKT3}

Double luciferase reporter gene system assay results showed that the luciferase activity was significantly lower in the co-transfected miRNA-320b mimics and pMIRREPORT Luciferase-AKT3-3' UTR (WT) cell group and miRNA-320b mimics and pMIR-REPORT LuciferaseAKT3-3' UTR (MT) cell group than in the other two groups (Fig. 6). These findings indicated that miRNA (hsa-miR-320b) can regulate the luciferase expression of the 3'UTR of the AKT3 gene $(P<0.0001)$. However, after mutating the binding site, there was no significant change in this regulatory relationship $(P=0.0507)$, suggesting that miRNA (hsa-miR-320b) does not regulate AKT3 expression through this binding site. 


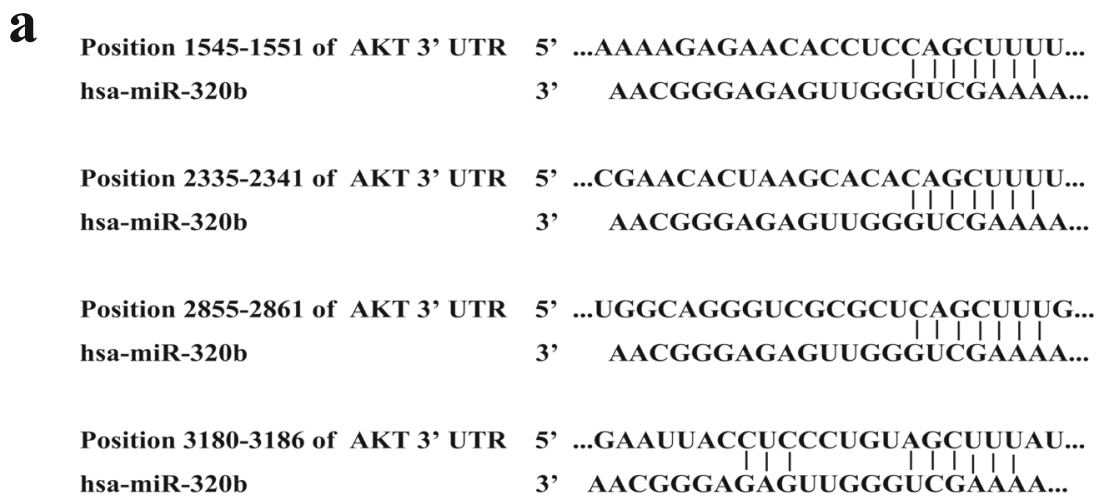

b

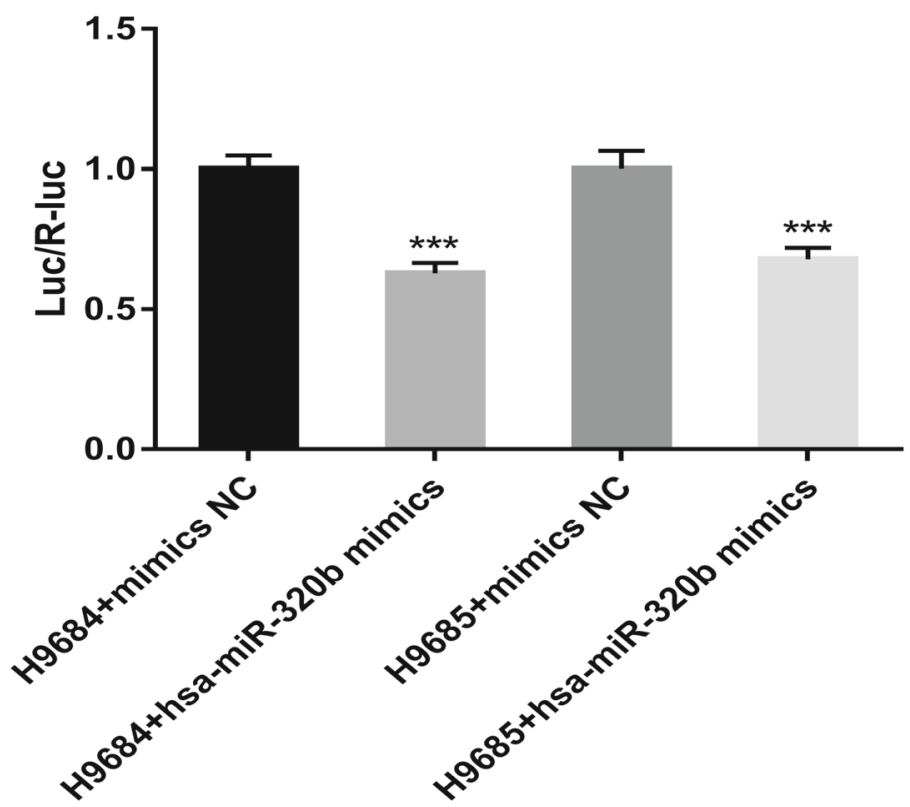

Fig. 6. Prediction of miR-320b binding site in AKT3 and miR-320b regulation on target gene (AKT3). a Predicting miR-320b binding site in AKT3 $3^{\prime} \mathrm{UTR}$. b Detection of miR-320b regulation on target gene AKT3 by double luciferase reporter assay. The miR-320b binding site on AKT3 was predicted by the targetscan software (available on http://www.targetscan.org). The results showed that the luciferase activity of the wild-type AKT3 3' UTR reporter vector was significantly inhibited compared to that of the control group $(P<0.0001)$. After mutating the predicted binding site, miR-320b still had a significant inhibitory effect on luciferase activity $(P<0.0001)$. There was no significant change in the regulatory relationship $(P=0.0507)$.

\section{Protein Levels of the Target Gene AKT3 in Transfected Cells Detected by Western Blot Analysis}

NHEKs were harvested $48 \mathrm{~h}$ after lentivirus transfection, and AKT3 levels were detected by Western blotting using GAPDH as an endogenous control. We found that AKT3 levels were higher in the hsa-miR-320b-KD group than in the NC group (Fig. 7). Next, we investigated other proteins related to AKT3-mediated intracellular signaling pathways and found that miR-320b knockdown in NHEKs significantly increased STAT3 and SAPK/JNK protein phosphorylation levels (Fig. 8).

\section{DISCUSSION}

Psoriasis is an immune-mediated, chronic inflammatory skin disease. The pathogenesis of this disease is related to heredity, environment, immunity, and other factors but has not yet been fully elucidated. miRNAs are short, noncoding RNAs that affect the post-transcriptional levels of target genes and have a role in many biological processes, such as growth, development, proliferation, and differentiation. Many studies have confirmed that there are significant differences in miRNA expression in patients with 
a

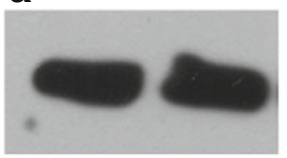

AKT3

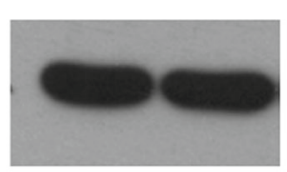

GAPDH

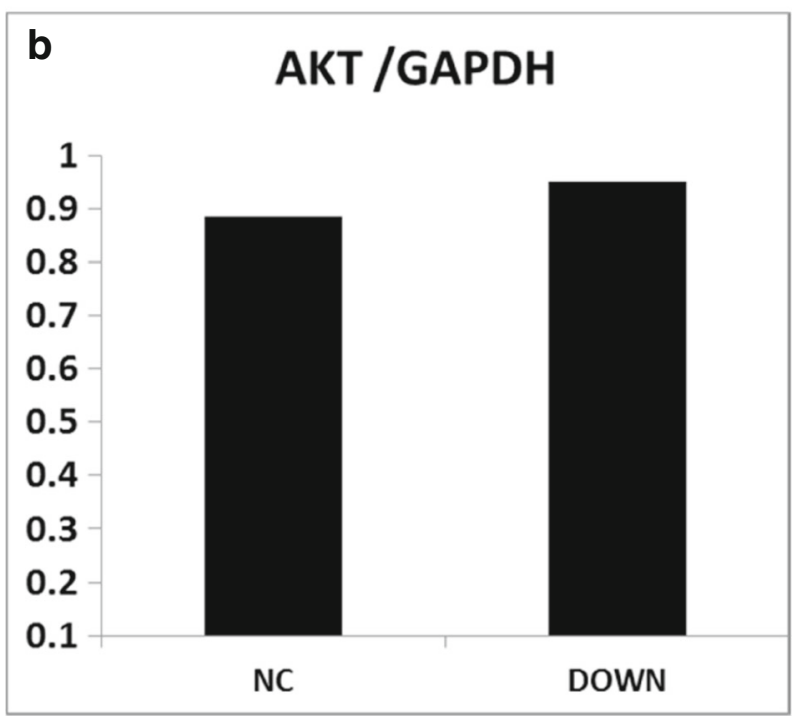

Fig. 7. Effect of hsa-mir-320b on AKT3 protein expression by western blotting. The level of AKT3 in the miR-320b-KD(DOWN) group was increased compared to that in the normal control (NC) group. GAPDH was used as an endogenous control. psoriasis, and miRNAs play an important role in the pathogenesis of psoriasis [6-12].

miR-320, a tumor suppressor gene, is a newly discovered miRNA that is significantly downregulated in a variety of tumors, including colorectal cancer, cervical cancer, and cholangiocarcinoma [15-20]. miR-320 not only inhibits the G1/S transition of the normal cell cycle [21] but also inhibits tumor cell proliferation [22]. It has been reported that miR-320 can be used as a tumor suppressor to inhibit fatty acid synthase (FASN) expression, which inhibits osteosarcoma cell proliferation [23]. Some scholars have found that miR-320 inhibits gastric cancer proliferation by targeting the FOXM1-P27KIP1 pathway [24]. That study found that miR-320b can inhibit colorectal cancer cell proliferation by targeting c-MYC directly to downregulate its mRNA and protein expression [25]. As aberrant miR-320 expression is found in many tumors, the role of miR-320 in disease development and its molecular mechanisms are also attracting attention. Psoriasis is a non-neoplastic disease characterized by the hyperproliferation of keratinocytes. A study by Wei et al. found that miR-320 expression is significantly lower in psoriatic lesions than in healthy control skin tissues; miR-320 may participate in psoriasis development by regulating the expression of its downstream target gene survivin [13]. This study also found that miR-320b is downregulated in the epidermal tissue of

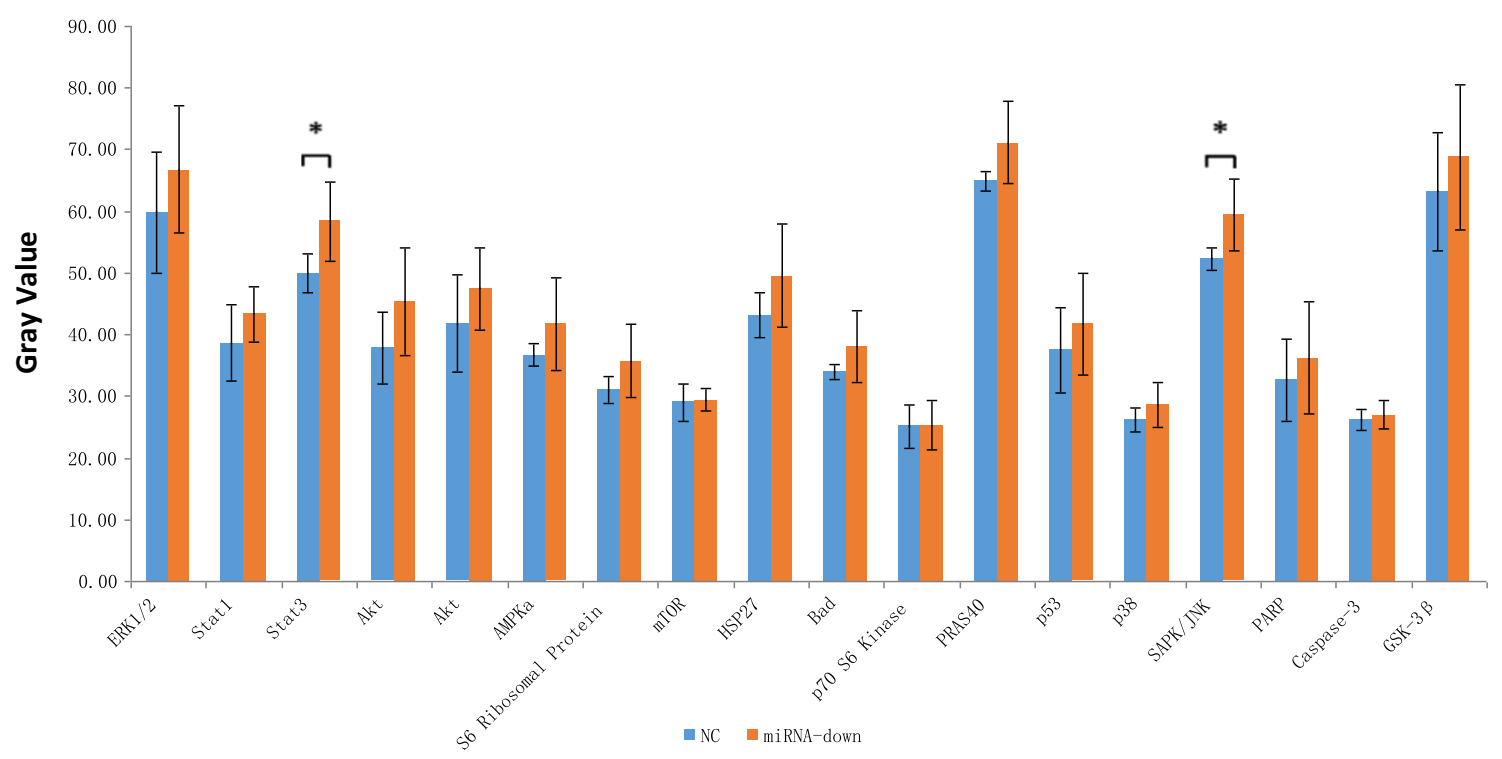

Fig. 8. Investigation of changes in the activation of the signaling pathway using the PathScan intracellular signaling array kit. Downregulation of miRNA$320 \mathrm{~b}$ was associated with increased protein phosphorylation in the STAT3 and SAPK/JNK pathways. NC normal control, miRNA-down miR-320bknockdown; *compared with negative control, $P<0.05$. 
psoriasis lesions. Downregulating miR-320b promotes keratinocyte proliferation.

AKT, also known as protein kinase B (PKB), is a serine/threonine protein kinase that was originally identified as a viral oncogene, V-akt homolog. The AKT family includes three subclasses, AKT1, AKT2, and AKT3. Changes in AKT expression and activity can lead to cell apoptosis, aberrant proliferation, and even cancer development [26]. Studies have shown that AKT1 and AKT2 expression in psoriatic lesions is not significantly different from that in normal skin. In contrast, AKT3 expression is significantly higher in psoriatic lesions than in normal skin tissue. These data suggest that increased AKT activity in psoriasis lesions may be related to increased AKT3 levels [27]. AKT is a direct protein target downstream of the phosphoinositide 3 kinase (PI3K) signaling pathway. Many studies have shown that the PI3K/AKT signal transduction pathway is an important pathway for cell survival; is involved in cell growth, cell proliferation, angiogenesis, cell energy metabolism, and tumor cell invasion; and plays an important role in cell growth and proliferation [28]. Studies have shown that AKT kinase activity is significantly higher in the lesions of patients with psoriasis than in normal skin. Increased AKT activity can cause excessive keratinocyte proliferation and dermal papillary hyperplasia [29].

Signal transducer and activator of transcription-3 (STAT3), which is an important transcriptional regulator, plays an important role in regulating cytokine networks, cytokine, and inflammatory mediator gene expression and immune responses and participates in the pathogenesis of psoriasis [30].

c-Jun N-terminal Kinase (JNK), also known as stress-activated protein kinase (SAPK), is a member of the mitogen-activated protein kinase (MAPK) family. JNK signaling pathways can be activated by a variety of factors, including cytokines, growth factors, and stress (such as ionizing radiation, heat shock, and oxidative damage). A large number of experiments suggest that JNK signaling pathways plays a crucial role in cell differentiation, apoptosis, stress responses, and the occurrence and development of various human diseases [31]. Studies have found that IL- $1 \alpha$ and IL$1 \beta$ produced by keratinocytes can stimulate fibroblasts to secrete keratinocyte growth factor (KGF) through the JNK substrate c-Jun, JunB transcription factors, and KGF released by fibroblasts. KGF released by fibroblasts stimulates the proliferation and differentiation of keratinocytes by paracrine signaling
[32]. Therefore, the JNK signaling pathway is also an important aspect of the pathogenesis of psoriatic cells and the relationship between keratinocytes and fibroblasts.

The regulatory mechanisms of miRNA genes in psoriasis are sophisticated. Multiple miRNAs can synergistically control a single gene. The same miRNA can also regulate multiple mRNA molecules, but most of the regulatory mechanisms remain unclear. Although many studies have found that the AKT3, STAT3, and SAPK/JNK signaling pathways play a role in the pathogenesis of psoriasis, the role and molecular mechanism of miR-320b in this disease are not clear enough and have not yet been studied. In this study, we found for the first time that miR$320 \mathrm{~b}$ was significantly downregulated in epidermal keratinocytes in psoriatic lesions. miR-320b could regulate the gene expression of AKT3. STAT3 and SAPK/JNK phosphorylation levels increased following miR-320b knockdown. This finding implies that miR-320b plays an important role in the pathogenesis of psoriasis in a Chinese Han population, which provides a novel diagnostic or therapeutic target for psoriasis. However, there are some limitations and unresolved issues, including a small sample size, in this study. Further experiments are needed to identify the miR-320b regulatory binding sites in AKT3. Then, the signaling pathways that they participate in can be mediated and applied in clinical trials.

\section{FUNDING}

This study was supported by the Shandong University Basic Research Business Funding Project (Grant no. 2014QLKY34).

\section{COMPLIANCE WITH ETHICAL STANDARDS}

Ethical Approval. This study was approved by the Research Ethics Committee of Qilu Hospital, Shandong University (No. KYLL 2015 108) and performed in accordance with the principles of the 1964 Declaration of Helsinki and its later amendments.

Informed Consent. Informed consent was obtained from all individual participants included in this study.

Conflict of Interest. The authors declare that they have no conflict of interest. 


\section{REFERENCES}

1. Parisi, Rosa, Deborah P.M. Symmons, Christopher E.M. Griffiths, and Darren M. Ashcroft. 2013. Global epidemiology of psoriasis: a systematic review of incidence and prevalence. Journal of Investigative Dermatology 133: 377-385.

2. Lowes, Michelle A., Anne M. Bowcock, and James G. Krueger. 2007. Pathogenesis and therapy of psoriasis. Nature 445: 866-873.

3. Tonel, Giulia, and Curdin Conrad. 2009. Interplay between keratinocytes and immune cells-recent insights into psoriasis pathogenesis. The International Journal of Biochemistry \& Cell Biology 41: 963-968.

4. Griffiths-Jones, Sam, Harpreet Kaur Saini, Stijn van Dongen, and Anton J. Enright. 2008. miRBase: tools for microRNA genomics. Nucleic Acids Research 36: D154-D158.

5. Taganov, Konstantin D., Mark P. Boldin, and David Baltimore. 2007. MicroRNAs and immunity: tiny players in a big field. Immunity 26: 133-137.

6. Meisgen, Florian, Ning Xu, Tianling Wei, Peter C. Janson, Susanna Obad, Oliver Broom, Nikoletta Nagy, Sakari Kauppinen, Lajos Kemény, Mona Ståhle, Andor Pivarcsi, and Enikö Sonkoly. 2012. MiR-21 is up-regulated in psoriasis and suppresses T cell apoptosis. Experimental Dermatology 21: 312-314.

7. Huang, Run-Yue, Li Li, Mao-Jie Wang, Xiu-Min Chen, Qing-Chun Huang, and Lu. Chuan-Jian. 2015. An exploration of the role of microRNAs in psoriasis: a systematic review of the literature. Medicine 94: e2030.

8. Ichihara, A., M. Jinnin, K. Yamane, A. Fujisawa, K. Sakai, S. Masuguchi, S. Fukushima, K. Maruo, and H. Ihn. 2011. microRNA-mediated keratinocyte hyperproliferation in psoriasis vulgaris. The British Journal of Dermatology 165: 1003-1010.

9. Wei, Tianling, Ning Xu, Florian Meisgen, Mona Ståhle, Enikö Sonkoly, and Andor Pivarcsi. 2013. Interleukin- 8 is regulated by miR-203 at the posttranscriptional level in primary human keratinocytes. European Journal of Dermatology. http:// www.jle.com/10.1684/ejd.2013.1997.

10. Primo, Maria Nascimento, Rasmus O. Bak, Beatrice Schibler, and Jacob Giehm Mikkelsen. 2012. Regulation of pro-inflammatory cytokines TNF $\alpha$ and IL24 by microRNA-203 in primary keratinocytes. Cytokine 60: 741-748.

11. Xu, Ning, Petter Brodin, Tianling Wei, Florian Meisgen, Liv Eidsmo, Nikoletta Nagy, Lajos Kemeny, Mona Ståhle, Enikö Sonkoly, and Andor Pivarcsi. 2011. MiR-125b, a microRNA downregulated in psoriasis, modulates keratinocyte proliferation by targeting FGFR2. The Journal of Investigative Dermatology 131: 1521-1529.

12. Lerman, Galya, Moran Sharon, Raya Leibowitz-Amit, Yechezkel Sidi, and Dror Avni. 2014. The crosstalk between IL-22 signaling and miR-197 in human keratinocytes. PLoS One 9: e107467.

13. Wei, Y.P., R.X. Zhang, S.J. Shi, et al. 2016. Expressions of miR-320 and its downstream target gene survivin in psoriatic lesions. Journal of Practical Dermatology 9: 225-228.

14. Zhao, Bian. 2010. Chinese clinical dermatology. 3rd edn. 1008 1112. Nanjing: Jiangsu Science and Technology Press (in Chinese).

15. Dong, Y., W.K. Wu, C.W. Wu, J.J. Sung, J. Yu, and S.S. Ng. 2011. MicroRNA dysregulation in colorectal cancer: a clinical perspective. British Journal of Cancer 104: 893-898.

16. Roth, Patrick, Jörg Wischhusen, Happold Caroline, P. Anoop Chandran, Silvia Hofer, Günter Eisele, Michael Weller, and Andreas
Keller. 2011. A specific miRNA signature in the peripheral blood of glioblastoma patients. Journal of Neurochemistry 118: 449-457.

17. Li, Xinhua, Feijun Luo, Qian Li, Meihua Xu, Deyun Feng, Guiying Zhang, and Wu. Wei. 2011. Identification of new aberrantly expressed miRNAs in intestinal-type gastric cancer and its clinical significance. Oncology Reports 26: 1431-1439.

18. Song, Tao, Wei Xia, Ningsheng Shao, Zhang Xu, Chunyang Wang, Yiguang Wu, Jun Dong, Wei Cai, and Hongzhao Li. 2010. Differential miRNA expression profiles in bladder urothelial carcinomas. Asian Pacific Journal of Cancer Prevention 11: 905-911.

19. Zhao, Hongchao, Taotao Dong, Houmin Zhou, Linlin Wang, Ao Huang, Bo Feng, Yingjun Quan, Runsen Jin, Wenpeng Zhang, Jing Sun, Daohai Zhang, and Minhua Zheng. 2014. miR-320a suppresses colorectal cancer progression by targeting Rac1. Carcinogenesis 35: 886-895.

20. Song, Cheng, Ting Zhang, Yao-Hui Xu, and Taihu Rehabilitation Hospital of Jiangsu Province, Jiangsu Provincial Research Center for Health Assessment and Intervention. 2016. Expression of new biomarker miR-320 in cervical cancer and its clinical significance. Maternal and Child Health Care of China 2: 238-240.

21. Duan, Huihan, Yiguo Jiang, Hongyu Zhang, and Yan Wu. 2010. MiR-320 and miR-494 affect cell cycles of primary murine bronchial epithelial cells exposed to benzo[a]pyrene. Toxicology In Vitro 24: 928-935.

22. Schaar, Dale G., Daniel J. Medina, Dirk F. Moore, Roger K. Strair, and Yi Ting. 2009. miR-320 targets transferrin receptor 1 (CD71) and inhibits cell proliferation. Experimental Hematology 37: 245255.

23. Zhen, C.C., Q.C. Xue, and T. Shi. 2014. MicroRNA-320 inhibits osteo-sarcoma cells proliferation by directly targeting fatty acid synthase. Tumor Biology 35: 4177-4183.

24. Wang, Y.Y., J.P. Zeng, J.Y. Pan, X. Geng, L. Li, J. Wu, P. Song, Y. Wang, J. Liu, and L. Wang. 2016. miR-320a inhibits gastric carcinoma by targeting activity in the Fox M1-P27 KIP1 axis. Oncotarget 7: 29275-29286.

25. Wang, H., F. Cao, X. Li, et al. 2015. miR-320b suppresses cell proliferation by targeting c-Myc in human colorectal cancer cells. BMC Cancer 15: 1-9.

26. Song, Gang, Gaoliang Ouyang, and Shideng Bao. 2005. The activation of Akt/PKB signaling pathway and cell survival. Journal of Cellular and Molecular Medicine 9: 59-71.

27. Man, Xiao-hong, Xiao-yan Zhang, Hong-yan Li, Bo Xu, Juan Tang, Yang-xin Chen, and Pan Lin. 2011. The expression of Akt1, Akt2, AKT3 in the lesions of psoriasis vulgaris. Chinese Journal of Dermatovenereology 25: 338-340.

28. Song, G., G. Ouyang, and S. Bao. 2005. The activation of Akt/PKB signaling pathway and cell survival[J]. Journal of Cellular and Molecular Medicine 9 (1): 59-71.

29. Zhang, Xiao-Yan, Ping Zhou, Li-Ping You, Chang-An Yu, Lin Pan, and Sheng-Qing Ma. 2009. Increased activities of AKT in psoriatic epidermis. Chin J Dermatol 42 (6): 413-416.

30. Sano, S., K.S. Chan, S. Carbajal, J. Clifford, M. Peavey, K. Kiguchi, S. Itami, B.J. Nickoloff, and J. DiGiovanni. 2005. Stat3 links activated keratinocytes and immunocytes required for development of psoriasis in a novel transgenic mouse model. Nature Medicine 11: 43-49.

31. Zenghui, L.I., and Liao Aijun. 2010. JNK signal pathway. International Journal of Pathology and Clinical Medicine 30 (3): 273-276.

32. Werner, S., and H. Smola. 2001. Paracrine regulation of keratinocyte proleferation and differentiation [J]. Trends in Cell Biology 11 (4): $143-146$. 